\title{
MEASURING THE NEAR-FIELD OF EXTRA-ORDINARY TRANSMISSION THROUGH A PERIODIC HOLE-ARRAY
}

\author{
M.W. Docter ${ }^{\mathrm{a} *}$, I.T. Young ${ }^{\mathrm{a}}$, O. Piciu, A. Bossche ${ }^{\text {b }}$ P.F.A. Alkemade ${ }^{\mathrm{c}}$, P.M. van den Berg ${ }^{\mathrm{a}}$ \\ and Y. Garini ${ }^{\mathrm{a}}$ \\ ${ }^{\mathrm{a}}$ Imaging Science and Technology, ${ }^{\mathrm{b}}$ Micro-Electronics and ${ }^{\mathrm{c}}$ Nanoscience departments \\ Delft University of Technology, Faculty of Applied Sciences, \\ Lorentzweg 1, Delft 2628 CJ, The Netherlands
}

* M.W.Docter@tnw.tudelft.nl; phone: +31-15-278-1303, fax: +31-15-278-6740

\begin{abstract}
The knowledge of the near-field of extra-ordinary transmission through hole-arrays is mostly theoretical; there is less experimental validation of the theory. We study the near-field properties by measuring fluorescent molecules that are immersed in a solution and their Brownian motion. The measurements are performed by filling the space above the hole-array with fluorescent solution and exciting these molecules through the hole-array. By measuring both the fluorescence and the direct exciting light, it is possible to learn about the near-field properties.
\end{abstract}

Keywords: plasmons, mid-field, nanostructure, hole array, near-field, high resolution, microscopy

\section{Introduction}

Extra-ordinary transmission (EOT) is a hot topic for its three qualities: (1) The transmission exceeds unity (2) Spectral selection of the transmitted light and (3) the narrow angular spread [1]. At first a lot of the research aimed at a better understanding of the way in which hole-array properties influence these qualities. The varied properties were holeshape, hole dimensions, period and material [2]. Besides gaining physical insight, the research interest in EOT now includes possible applications in which EOT plays the key role. The spectral selection makes EOT suitable for filtering purposes [3], the high transmission and angular spread for microscopy [4] and lithography [5].

We proposed a concept for a new microscope we termed the mid-field microscope [6]. The transmitted nearfield will illuminate a biological sample stained with fluorescent molecules and the fluorescent light is collected in farfield. In order to fully understand and interpret the images recorded in far-field, it is crucial to know the near-field. Although theories have been developed (Section 2) they are usually based on rigorous solutions of Maxwell's equations and therefore less physical insight can be obtained. We therefore proposed a new theory based on Green's theorem and on an analytical solution of the field on the surface. The rigorous solution allows us to distinguish the contribution of the direct transmitted light from that of the surface plasmons, an ongoing controversy in the field. In addition, the calculations can be validated by using the mid-field microscope set-up and a solution of fluorescent molecules to measure the near-field of EOT. By conducting this experiment we actually are obtaining a proof of concept of our microscope.

We first introduce our theory and provide an overview of the predicted EOT field, both by others and ourselves. An overview of indirect measurements on the transmitted near-field will be provided as well. We will then introduce the concept and set-up of the mid-field microscope. Finally, preliminary experimental results will be shown and discussed.

Nanophotonics, edited by David L. Andrews, Jean-Michel Nunzi, Andreas Ostendorf, Proc. of SPIE Vol. 6195, 61951L, (2006) - 0277-786X/06/\$15 - doi: 10.1117/12.661969 


\section{Predicting the near/far-field of EOT}

The theories that were developed for analyzing the transmission through hole-arrays are based on solving the Maxwell's equations with valid boundary conditions. A lot of the simulations are using finite time domain calculations, in which local Maxwell's equations are solved. Though accurate, these calculations do not allow the separation of the influence of surface plasmons, which most researchers suppose to be the cause of EOT, from that of the direct transmission. However, it does show important and interesting features which can be used to derive an easier-to-understand theory. For example, since we are interested only in the field distribution of the transmitted light, we can use the results derived by Martín-Moreno [7] who found that the angular distribution only depends on the exit surface. This fact simplifies the calculation and allows the development of a theory that limits the treatment to the half-space that starts at the exit surface. The result of such theory should be consistent with other results, like the one that shows light originating from the non-illuminated part of the exit surface [8] or interference fringes in between transmission through slits [9].

The model we developed can be used for either a slit of holes, that we refer to as a two dimensional model (2D) or for a hole array, which requires a three-dimensional (3D) solution. This model is only valid for two dimensions, but for 3D the polarization decomposes in linear polarization along the principal axes, for which the hole-array acts like an array of slits [10]. The preferential polarization is along the diagonal of the hole-array. Therefore we expect the upcoming $3 \mathrm{D}$ experimental results to be comparable to our $2 \mathrm{D}$ model by taking the polarization into account.

Our (2D) theory makes use of the Green formalism; this allows us to calculate the field at any point in space if the field at a surface surrounding this point is known. Only the field at the exit surface of the array is taken into account. Because the solution is based on an analytical solution to the field on the array-surface, we can separate the influences of the direct transmission and the surface plasmons on the exit surface by separating the contributing fields. The directly transmitted field at the exit of the slit is assumed to be uniform due to the small dimensionality of the hole. The surface plasmons contribute to a fluctuating cosine field on the metal surface; therefore the wavelength dependent on the size of the metal surface (period $a$ minus diameter hole $d$ ). Note that in the case of a 3D hole-array $a-d$ might be replaced by $a \sqrt{ } 2-d$ due to chosen polarization in the diagonal direction. This result have a simple and intuitive physical interpretation, and it is more accurate than models that uses the period of the hole array [1], because inside the holes no surface plasmons will exist. Our model is described by the following equations, in which $H_{0}^{(1)}$ is the Hankel function of the first kind and zero order and $k_{\mathrm{a}}$ is the wave number in air, $\mathrm{H}$ is the magnetic field on the exit surface of the hole-array and $k_{\mathrm{a}}$ is the wave vector in air:

$$
\begin{aligned}
& H(x, z)=\frac{-i}{2} \int H\left(x^{\prime}, 0\right) \frac{\partial}{\partial z} \mathrm{H}_{0}^{(1)}\left(k_{\mathrm{a}} r\right) d x^{\prime} \quad z \geq 0 \\
& H(x, z \geq 0)=\left\{\begin{array}{lc}
h_{y} \hat{y} & -a / 2 \leq x<-(a-d) / 2 \\
h_{y} \cos \left(k_{x} x\right) \hat{y} \exp \left[i\left(k_{z} z-\omega t\right)\right] & -(a-d) / 2 \leq x<(a-d) / 2 \\
h_{y} \hat{y} & (a-d) / 2 \leq x<a / 2
\end{array}\right.
\end{aligned}
$$

By using this and the Green function formalism, the field above the array is calculated and the result is shown in figure 1. This is calculated for the case of an Au slit-array consisting of 25 slits, a hole-diameter of $150 \mathrm{~nm}$ and a period of $600 \mathrm{~nm}$ ). 

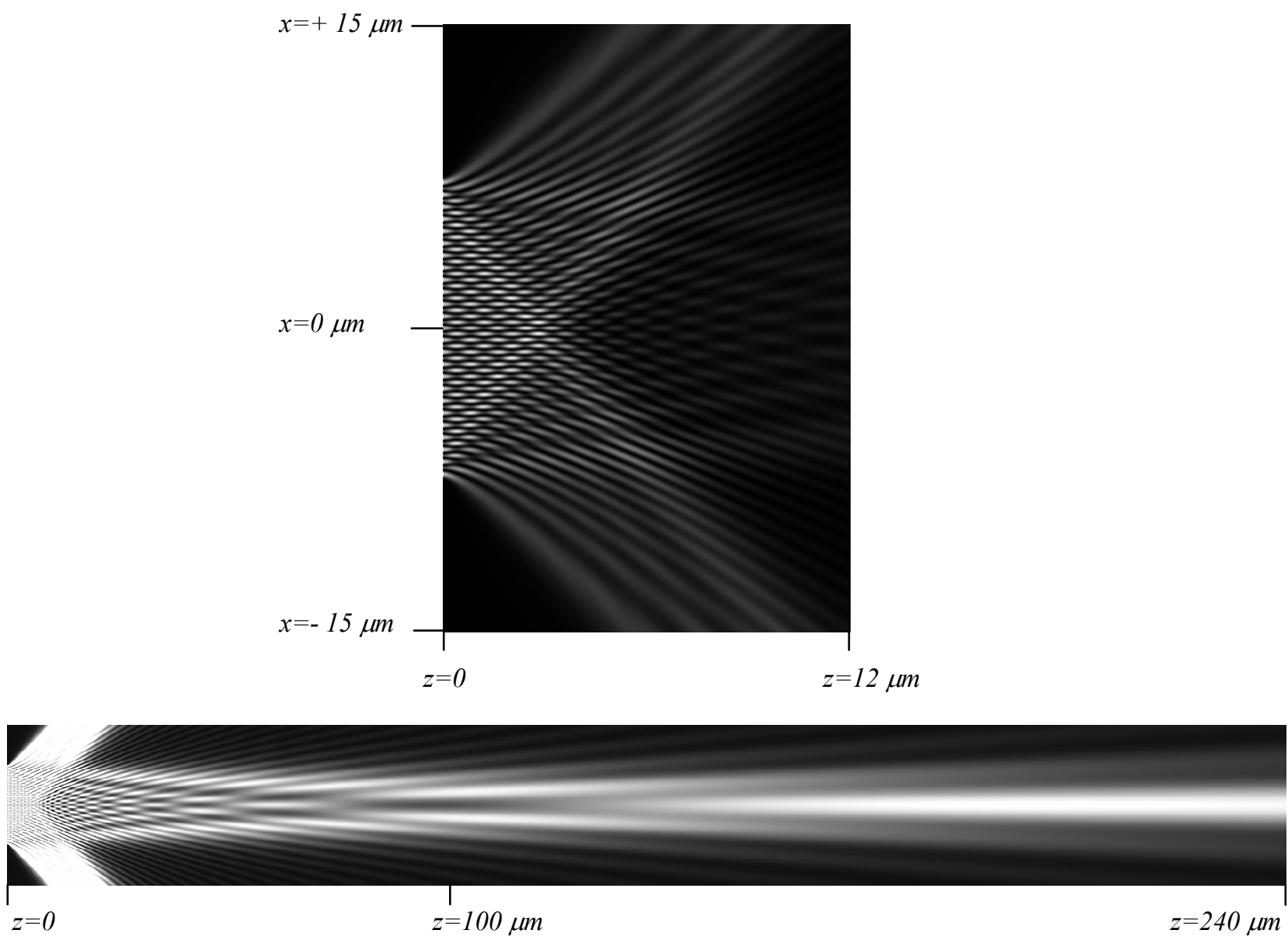

Figure 1. The near-field (upper) and far-field (lower) transmission through 25 slits, with width $150 \mathrm{~nm}$ and period $600 \mathrm{~nm}$. The color scale is linear; the lower image is over-scaled (x10) in order to see the far-field. The height of both images is $30 \mu \mathrm{m}$, the widths of the upper and lower images are 12 and $240 \mu \mathrm{m}$ in correspondence.

By repeating the calculations for the cases with transmission and either with or without surface plasmons for a slit array or a single large slit (with same width as the array), the following results are found:

1. When taking the contribution of surface plasmons into account, the intensity of the field is three times as high as when only transmission is used without surface plasmons.

2. The near-field reveals an interference pattern with features smaller than the wavelength of light, especially along the $\mathrm{x}$-axis (parallel to the surface).

3. The width and height above the surface of the sub-wavelength near-field pattern depends on the number of slits; it extends the furthest above the center slit (see figure 1 top).

4. The far-field transmission through the array resembles the transmission through a single large slit, except for the $1^{\text {st }}$ order diffraction which is only present at transmission through the array.

5. The surface plasmons contribute mostly to the first order diffraction

When performing an experiment to measure the pattern of the field, the results depend on the numerical aperture that is used for collecting the transmitted light. Spatial information of the array can be lost, especially when the first order diffraction is not captured by the objective lens (due to a low numerical aperture). This results in a measurement that only captures the zero-order diffraction (figure 1 bottom) and a low angular spread will be measured, which is likely to be the case for the measured angular spread of $\pm 3^{\circ}[11]$. 


\section{Measuring the near-field}

When measuring the transmission in the far-field, no information can be gathered about the near-field. One measures the light as arriving in far-field, but the near-field pattern is lost. It is still possible to obtain some information on the near-field if the far-field measures the fluorescence of molecules that are excited by the transmitted light as suggested in $[6,12]$ or Cs atoms used in [13]. It is also possible to measure directly in the near-field, like with lithographic methods. Using a negative resist allows the mapping of the near-field [14]; different intensity levels were found when using different exposure times. Afterwards the developed resist is observed with an electron microscope, but unfortunately only transmission through a single hole was measured. The intensity through an array is measured on a positive resist, but then only the first layer of the resist is used (light cannot penetrate that deep?) [5].

We propose to use fluorescent molecules to probe the near-field of transmitted light [12]. If these molecules are diluted in a fluid, they move by Brownian motion. The travel of the beads depends on the diffusion coefficient, which itself depend on the inverse of the viscosity and the radius of the bead [15]. Since Brownian motion is a random motion, depending on time, viscosity and radius of the probes the total area of sight will be covered.

\section{Experimental setup}

We use the setup of the mid-field microscope, shown in figure 2. An Argon-ion laser beam (514 nm, LaserOptics) illuminates an array under a nominal incidence angle of $0^{\circ}$. The array consists of holes made in a layer of $200 \mathrm{~nm}$ of gold, which is evaporated onto a glass substrate with a $2 \mathrm{~nm}$ titanium bond layer in between the gold and the glass. The circular holes are made with a Focused Ion Beam machine; the period is $600 \mathrm{~nm}$ and the diameter $150 \mathrm{~nm}$. The array can be moved upwards to the sample with a vertical linear stage (Newport).

The sample stained with fluorescent molecules can be either in vivo or in vitro. The fluorescent molecules act as probes of the local (near-) field and can be observed in far-field. This is in contrast to observing the transmission through array and sample directly in far-field where the transmission through the array and the propagation afterwards are indistinguishable. The sample is positioned on top of the array; it is moved with piezo precision (Physik Instrumente) under observation with an epi-fluorescence microscope (BXFM, Olympus). Since the illumination is not continuous, the piezo is used to scan the sample in the focal plane of the lens. The objective lens (UPLFLN100xOI2, Olympus) is an oil immersion lens with a variable numerical aperture (NA) between 0.6 and 1.3. The NA is adjusted by tuning a variable iris diaphragm. The working distance is 100 micrometer, which allows to collect the fluorescent from ranges which are very close to the array-surface (the position of the lens is approximately $100 \mu \mathrm{m}$ from the holearray, see Fig. 1 bottom). The objective is moved by another piezoelectric stage along the optical axis (PIFOC, Physik Instrumente) in order to enable a 3D scan of the sample. A sensitive CCD (ORCA-AG, Hamamatsu) acquires an image each time after one of the piezo stages was moved. After acquisition, the information that contains many images captured at many different focal planes is combined and processed with a special image-processing algorithm that we developed and that allows us to reconstruct the three-dimensional image. 


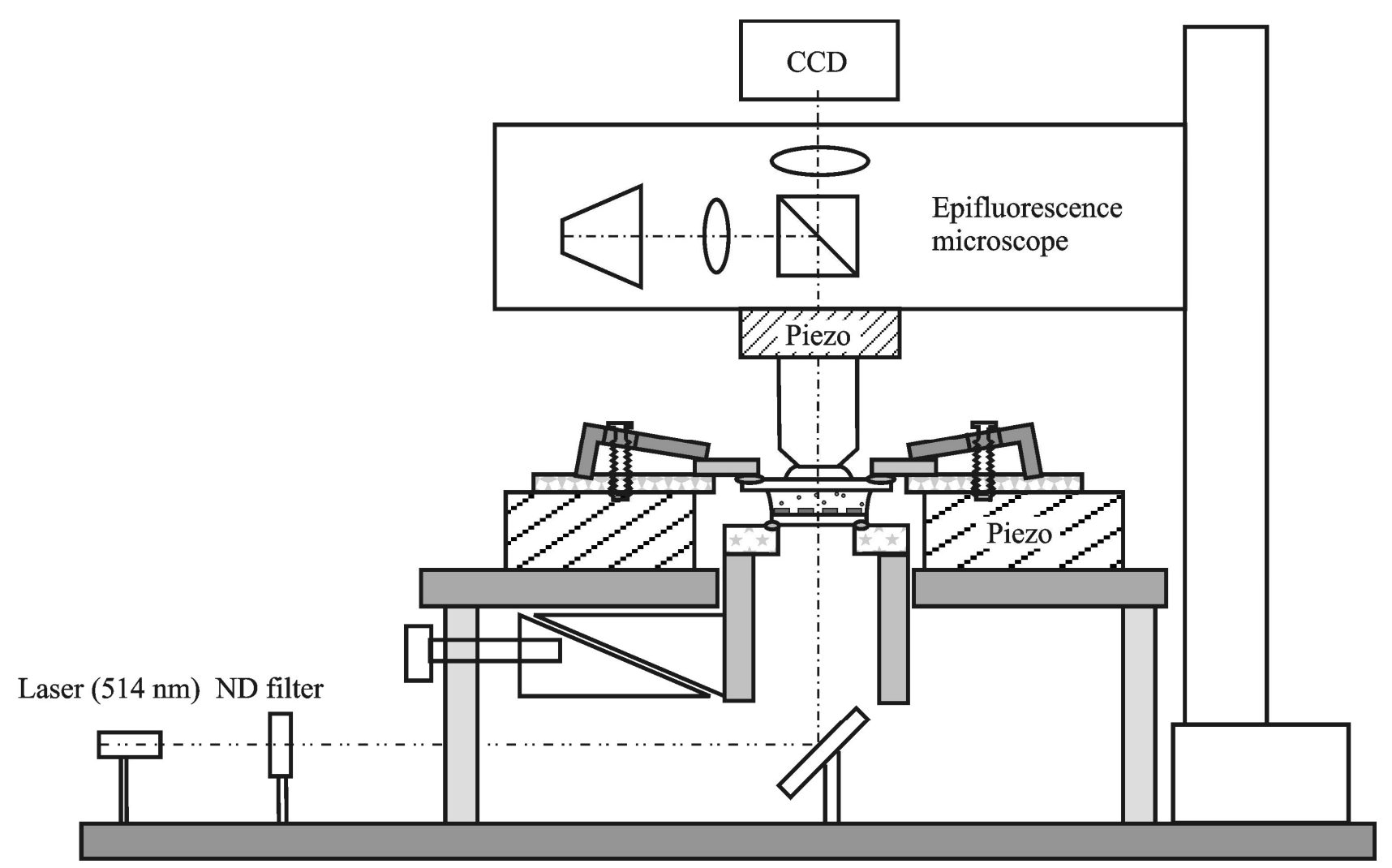

Figure 2. The mid-field set-up. Laser light is transmitted through the array, illuminating the sample, which in this case is a fluid with fluorescent molecules. These molecules in the sample work as probes for the transmitted field and their emission light is collected by the CCD.

In order to measure the transmitted near-field, we use a fluid with a given concentration of fluorescent molecules that is dropped on top of the array. The molecules will move by Brownian motion in the liquid. If the concentration is high enough, it can be assumed that the distribution of the fluorescent molecules is uniform in the whole fluidic volume above the hole-array. In addition, many images can be acquired in order to average-out un-even local concentration of the fluorescent molecules.

Rhodamine $6 \mathrm{G}(10 \mathrm{mM})$ which has a typical size of a few nanometers was used in water with a typical acquisition time of $10 \mathrm{~ms}$. The fluorescence is blurred with the point spread function (PSF) of the system. Therefore we should deconvolve our images with the PSF obtained either theoretically [16] or experimentally by making a 3D images of a small bead or hole. 


\section{Results}

The array was first aligned with the system with a 20x magnification objective lens under reflection from the side and later with the 100x lens under transmission of laser light. The expectation based on our theoretical predictions was, that we would not see much details related to the spatial distribution of holes. This was true for the low NA of 0.6, but surprisingly the details of the individual holes became clearer when increasing the NA. This is shown in figure 3.
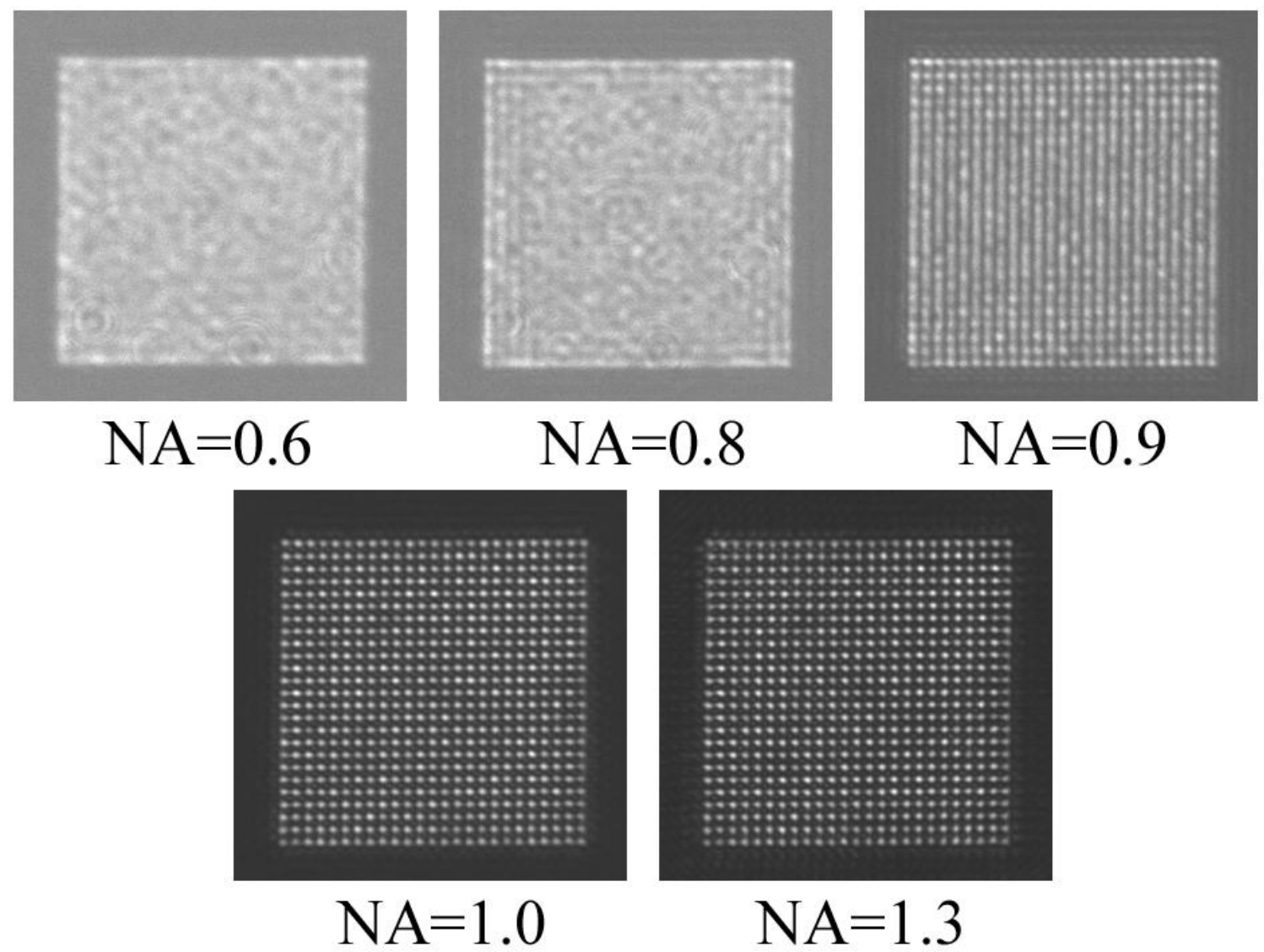

Figure 3. Transmission through a $25 * 25$ hole-array, with water on top, a cover slip and immersion oil. The same wavelength as used for illumination is measured. The numerical aperture (NA) of the objective lens is changed from 0.6 to 1.3 .

The image of the array shows almost no detail for low NA but it does for high NA. The transition between an image with and without details of the array lies around $\mathrm{NA}=0.9$. This suggests that around this angle a diffraction order appears, which contributes to a sharp image. Earlier results from a measurement in far-field in air (so in between array and lens nothing but air) [6], showed that for a dry lens with NA=0.75 no details appeared. During the previous drylens experiment, the NA was not high enough to capture the diffraction.

We predicted that there will be no details in the image of the direct transmission. However, we observed a clear image with distinguishable holes (Fig. 3). This may have two possible explanations. The first explanation may be due to the coverage and filling of the holes by water. If the holes are not totally filled with water, the water will have a meniscus shape: the surface tension is much larger than gravitational forces (Low Reynolds number), this tension pulls the surface into a sphere while minimizing the surface area [17]. A spherical surface between air and water has a focusing effect, light will be refracted and when imaging this light it will appear to originate from a virtual source point above the holes. 
Our setup is also equipped with epi-fluorescence microscope and allows one to illuminate the fluorescent molecules in reflection mode with a mercury lamp. However, when using epi-illumination, the beads may also be excited by light that is reflected from the gold surface. In this case, the fluorescence intensity above the holes should be dimmer than the fluorescence above the metal surface. If the holes are not filled, the intensity should be approximately less than half of the normal value (due to no reflection outside the metal, which implies no reflection in the holes). The result is shown in figure 5; the intensity of the fluorescence inside the holes is found to be approximately $95 \%$ of the intensity on the surface. Therefore, we cannot conclude whether the holes are filled with water or not.

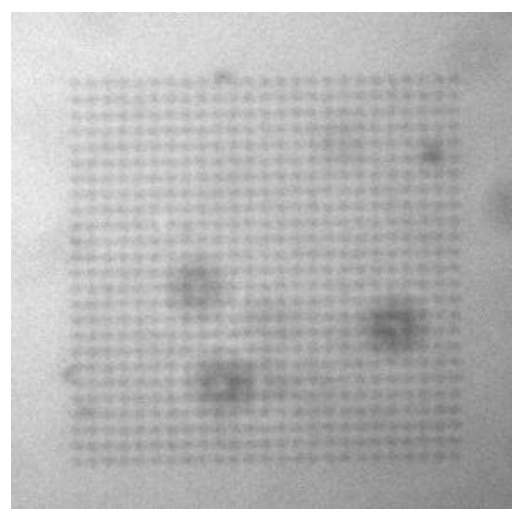

Figure 5. The array with on top a solution of Rhodamine in water $(0.1 \mathrm{mM})$ seen in epi-fluorescence.

The second explanation is a combination of the mismatch of the incoming wavelength and peak transmission and random polarization. As explained in section 2 the wavelength coupling in 2D and 3D array is different; the sizes of the metal surface are respectively $a-d$ and $a \sqrt{ } 2-d$. The wavelength of the laser $(514 \mathrm{~nm})$ corresponds to $a-d$, while the coupling is along $a \sqrt{2}-d$. In future experiments we will introduce a polarizer, the polarization being transmitted corresponds to the direction along the array (and not diagonally).

To prove that it is possible to measure the fluorescent signal even if the illumination is not ideal, we switch from observing the direct transmission to observing the fluorescent signal. It is a dim signal, since we are far away from the optimal transmitted wavelength, but after acquisition for 100 seconds the image shows distinct holes (Figure 6).
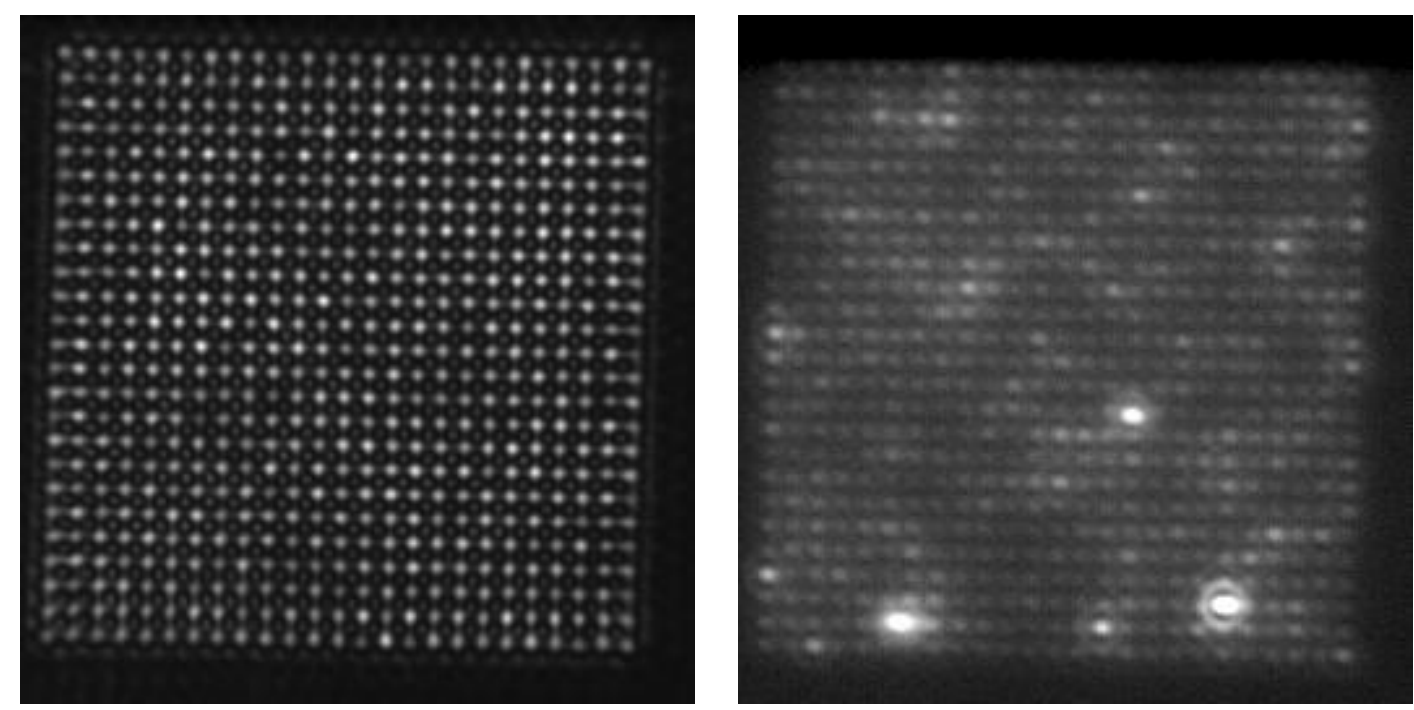

Figure 6. (left) direct transmission through the hole-array, single acquisition for 0.001 milliseconds; (right) fluorescent signal excited by the direct transmission after acquisition of 100 seconds. 


\section{Discussion \& Conclusion}

Knowledge about the near-field of EOT is vital to the feasibility of our mid-field microscope. The theoretical 2D model predicted an interference-like intensity distribution in the near-field. From control measurements, in which the direct transmission was measured, we noted that we did see distinct holes as predicted for NA $>0.9$. This is probably caused by a combination of 1. Partial filling of the holes and 2. Mismatch between the polarization and/or the wavelength of the incoming light and the dimensions of the hole-array. Unfortunately we could not validate the first possible cause by observing the fluorescent signal in reflection mode. Still we managed to measure a fluorescent signal after transmission of light through the hole-array; the image revealed the small features which were also present in the direct transmission

In future experiments we plan to treat the metal surface to ensure that the holes are filled with the fluid. Also we will use a polarizer to ensure the incoming light is polarized along the right axis of the hole-array. The experiments can be made more accurate by using a higher dilution in viscous fluid, which allows observation of single fluorescent molecules, since then the intensity at certain point can be directly derived from the image (instead of using deconvolution). Then the motion of the bead during the exposure time needed to acquire enough signal might be limiting the resolution.

It is definitely clear that there is a need for measurements of extra-ordinary transmission in near-field. More efforts should be spent for measuring the near-field by using fluorescent molecules and study the actual pattern.

\section{References}

1 T. W. Ebbesen, H. J. Lezec, H. F. Ghaemi, et al., Nature 391, 667 (1998).

2 H. F. Ghaemi, T. Thio, D. E. Grupp, et al., Physical Review B 58, 6779 (1998).

3 Y. Wang, Applied Physics Letters 82, 4385 (2003).

4 Smolyaninov, II, J. Elliott, A. V. Zayats, et al., Physical Review Letters 94 (2005).

5 W. Srituravanich, N. Fang, C. Sun, et al., Nano Letters 4, 1085 (2004).

6 M. W. Docter, I. T. Young, V. G. Kutchoukov, et al., SPIE Proceedings 5703, 118 (2005).

7 L. Martin-Moreno, F. J. Garcia-Vidal, H. J. Lezec, et al., Physical Review Letters 90, 167401 (2003).

8 S. C. Hohng, Y. C. Yoon, D. S. Kim, et al., Applied Physics Letters 81, 3239 (2002).

9 P. K. Wei, H. L. Chou, C. H. Wei, et al., Optics Communications 253, 198 (2005).

10 E. Altewischer, M. P. van Exter, and J. P. Woerdman, Journal of the Optical Society of America B-Optical Physics 20, 1927 (2003).

11 H. J. Lezec, A. Degiron, E. Devaux, et al., Science 297, 820 (2002).

12 B. Hecht, Philosophical Transactions of the Royal Society of London Series a-Mathematical Physical and Engineering Sciences 362, 881 (2004).

13 G. Gay, B. V. de Lesegno, R. Mathevet, et al., Applied Physics B-Lasers and Optics 81, 871 (2005).

14 B. Dragnea, J. M. Szarko, S. Kowarik, et al., Nano Letters 3, 3 (2003).

15 B. Rieger, H. R. C. Dietrich, L. R. van den Doel, et al., Microscopy Research and Technique 65, 218 (2004).

16 M. Born and E. Wolf, Principles of Optics : Electromagnetic Theory of Propagation, Interference and Diffraction of Light (Cambridge University Press, Cambridge, 1999).

17 B. Rieger, L. R. van den Doel, and L. J. van Vliet, Physical Review E 68 (2003). 